\title{
REGULAR FACTORIZATIONS OF CONTRACTIONS
}

\author{
BÉLA SZ.-NAGY AND CIPRIAN FOIAS,
}

\begin{abstract}
Equivalent conditions are given for the regularity of a factorization of a contraction, two of which exhibit immediately the duality property of this notion.
\end{abstract}

The concept of regular factorization of contractions of Hilbert spaces ${ }^{1}$ was introduced in [1] by the authors in connection with their investigations on the invariant subspace problem; cf. [2, §VII.3].

Let $A_{0}$ be a contraction of a Hilbert space $\mathfrak{A}$ into a Hilbert space $\mathfrak{A}_{*}$, and let

$$
A_{0}=A_{2} A_{1}
$$

be a factorization of $A_{0}$ as a product of a contraction $A_{1}$ of $\mathfrak{A}$ into some "intermediate" Hilbert space $\mathfrak{B}$, and of a contraction $A_{2}$ of $\mathfrak{B}$ into $\mathfrak{A}_{*}$ :

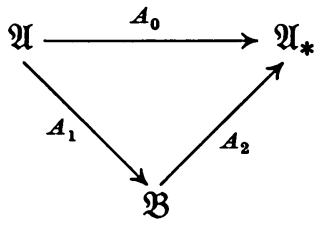

Define the corresponding "defect operators" by

$$
D_{j}=\left(I_{j}-A_{j}^{*} A_{j}\right)^{1 / 2}, \quad D_{* j}=\left(I_{* j}-A_{j} A_{j}^{*}\right)^{1 / 2} \quad(j=0,1,2),
$$

where $I_{j}$ and $I_{* j}$ denote the identity operators on the space of definition of $A_{j}$ and $A_{j}^{*}$, respectively.

The factorization (F) was defined to be regular if condition (i) given in the Proposition below holds. Some basic arithmetical properties of such factorizations were established in [2], in particular it was proven that $(F)$ is regular if and only if its dual

$$
A^{*}=A_{1}^{*} A_{2}^{*}
$$

Received by the editors December 15, 1972.

AMS (MOS) subject classifications (1970). Primary 47A50; Secondary 47A15.

Key words and phrases. Hilbert space, contraction, factorization, defect operators, dual factorization.

1 The Hilbert spaces considered are either all real or all complex. Separability is not assumed. Contraction means linear operator of norm $\leqq 1$.

(c) American Mathematical Society 1974 
is regular. This property does not follow easily from condition (i). This makes it desirable to find conditions equivalent to (i) and exhibiting the duality property. We are going to give two such conditions (ii) and (iii); the equivalence of (i) and (iii) was already stated (without proof) by Švarcman [3]. ${ }^{2}$

Proposition. For the factorization (F) the following three conditions are equivalent:

$$
\begin{aligned}
\overline{\left\{D_{2} A_{1} a \oplus D_{1} a: a \in \mathfrak{A}\right\}} & =\overline{D_{2} \mathfrak{B}} \oplus \overline{D_{1} \mathfrak{A}}, \\
\left\{\overline{\left.D_{2} b \oplus D_{*_{1}} b: b \in \mathfrak{B}\right\}}\right. & =\overline{D_{2} \mathfrak{B}} \oplus \overline{D_{* 1} \mathfrak{B}}, \\
D_{\mathbf{2}} \mathfrak{B} \cap D_{*_{1}} \mathfrak{B} & =\{0\} .
\end{aligned}
$$

The proof will be given in the steps (i) $\Rightarrow$ (ii) $\Rightarrow$ (iii) $\Rightarrow$ (i).

(1) We make use of the decomposition $\left(D_{* 1} \mathfrak{B}\right)^{-}=\left(A_{1} D_{1} \mathfrak{A}\right)^{-} \oplus \operatorname{ker} A_{1}^{*}$ generated by the contraction $A_{1}$; cf. [2, formula I.3.7]. Observe that for any $b \in \operatorname{ker} A_{1}^{*}$ we have $b=D_{* 1} b$, and hence

$$
0 \oplus b=\left(D_{2} b \oplus D_{* 1} b\right)-\left(D_{2} b \oplus 0\right) .
$$

As we obviously have $D_{2} b \oplus 0 \in\left(D_{2} \mathfrak{B}\right)^{-} \oplus\left(A_{1} D_{1} \mathfrak{2}\right)-$, we conclude that condition (ii) holds if (and only if) every element of the form $D_{2} b \oplus$ $A_{1} D_{1} a(a \in \mathfrak{A}, b \in \mathfrak{B})$ is the limit of a sequence of elements of the form $D_{2} b_{n} \oplus D_{* 1} b_{n}\left(b_{n} \in \mathfrak{B}\right)$. Now if condition (i) is satisfied then there exists a sequence $a_{n} \in \mathfrak{A}$ such that

$$
D_{2} A_{1} a_{n} \rightarrow D_{2} b \text { and } D_{1} a_{n} \rightarrow D_{1} a .
$$

Hence, setting $b_{n}=A_{1} a_{n}(\in \mathfrak{B})$ we have

$$
\begin{aligned}
D_{2} b_{n} \oplus D_{* 1} b_{n} & =D_{2} A_{1} a_{n} \oplus D_{* 1} A_{1} a_{n}=D_{2} A_{1} a_{n} \oplus A_{1} D_{1} a_{n} \\
& \rightarrow D_{2} b \oplus A_{1} D_{1} a .
\end{aligned}
$$

Therefore, (i) implies (ii).

(2) Let us notice that for any selfadjoint operator $S$ on a Hilbert space $\mathfrak{H}$ we have $\mathfrak{H}=(S \mathfrak{H})^{-} \oplus \operatorname{ker} S$. Hence it follows that $S \mathfrak{H}=S\left((S \mathfrak{H})^{-}\right)$. Thus we have in particular $D_{2} \mathfrak{B}=D_{2}\left(\left(D_{2} \mathfrak{B}\right)^{-}\right)$and $D_{* 1} \mathfrak{B}=D_{* 1}\left(\left(D_{* 1} \mathfrak{B}\right)^{-}\right)$. Hence, for every element $x \in D_{2} \mathfrak{B} \cap D_{* 1} \mathfrak{B}$ there exist elements $b^{\prime} \in\left(D_{2} \mathfrak{B}\right)^{-}$ and $b^{\prime \prime} \in\left(D_{* 1} \mathfrak{B}\right)-$ such that $x=D_{2} b^{\prime}=D_{* 1} b^{\prime \prime}$. Set $y=b^{\prime} \oplus\left(-b^{\prime \prime}\right)$. Clearly $y$ belongs to $\left(D_{2} \mathfrak{B}\right)^{-} \oplus\left(D_{* 1} \mathfrak{B}\right)^{-}$. Moreover, it is orthogonal to all elements

\footnotetext{
${ }^{2}$ AdDed IN PROOF (October 31, 1973). In the meantime the proof appeared in a paper of the same title in Mat. Issled. 8 (1973), 201-216, at p. 210. This proof of the implication (iii) $\Rightarrow$ (i) is identical with ours. However, the equivalent form (ii) does not occur in Šarcman's paper.
} 
of the form $D_{2} b \oplus D_{* 1} b(b \in \mathfrak{B})$, because

$$
\begin{aligned}
\left(y, D_{2} b \oplus D_{* 1} b\right) & =\left(b^{\prime}, D_{2} b\right)-\left(b^{\prime \prime}, D_{* 1} b\right) \\
& =\left(D_{2} b^{\prime}-D_{* 1} b^{\prime \prime}, b\right)=(x-x, b)=0 .
\end{aligned}
$$

Thus if (ii) is satisfied we must have $y=0$, and hence $b^{\prime}=0, b^{\prime \prime}=0$; consequently $x=0$.

Therefore, (ii) implies (iii).

(3) Let $v \oplus u$ be any element of $\left(D_{2} \mathfrak{B}\right)^{-} \oplus\left(D_{1} \mathfrak{A}\right)-$, orthogonal to all elements of the form $D_{2} A_{1} a \oplus D_{1} a(a \in \mathfrak{A})$. This means that

$$
A_{1}^{*} D_{2} v+D_{1} u=0
$$

hence we deduce

$$
A_{1} A_{1}^{*} D_{2} v+A_{1} D_{1} u=0, \quad D_{2} v-D_{* 1}^{2} D_{2} v+D_{* 1} A_{1} u=0
$$

so that

$$
D_{2} v=D_{* 1}\left(D_{* 1} D_{2} v-A_{1} u\right) .
$$

If (iii) is satisfied then $(\beta)$ implies $D_{2} v=0$; as $v \in\left(D_{2} \mathfrak{B}\right)^{-}$we conclude that $v=0$. Then $(\alpha)$ implies $D_{1} u=0$, and again since $u \in\left(D_{1} \mathfrak{A}\right)^{-}$, we conclude that $u=0$. Thus $v \oplus u$ is zero.

Therefore, (iii) implies (i).

This finishes the proof.

\section{REFERENCES}

1. B. Sz.-Nagy and C. Foiaş, Une caractérisation des sous-espaces invariants pour une contraction de l'espace de Hilbert, C.R. Acad. Sci. Paris, 258 (1964), 3426-3429. MR 29 \#1537.

2. —_, Analyse harmonique des opérateurs de l'espace de Hilbert, Masson, Paris; Akad. Kiadó, Budapest, 1967; English rev. transl., North-Holland, Amsterdam; American Elsevier, New York; Akad. Kiadó, Budapest, 1970. MR 37 \#778; 43 \#947.

3. Ja. S. Svarcman, On invariant subspaces of a dissipative operator and the divisors of its characteristic function, Funkcional. Anal. i Priložen. 4 (1970), no. 4, 85-86= Functional Anal. Appl. 4 (1970), 342-343. MR 43 \#3832.

Bolyai Institute, University of SZeged, Szeged, Hungary

Mathematics Institute, Academy of Sciences, Bucharest, Romania 